Draft Version November 11, 2018

Preprint typeset using $\mathrm{IATEX}_{\mathrm{E}}$ style emulateapj v. 08/13/06

\title{
MOND AND THE UNIVERSAL ROTATION CURVE: SIMILAR PHENOMENOLOGIES
}

\author{
Gianfranco Gentile ${ }^{1,2}$
}

Draft version November 11, 2018

\begin{abstract}
The Modified Newtonian Dynamics (MOND) and the Universal Rotation Curve (URC) are two ways to describe the general properties of rotation curves, with very different approaches concerning dark matter and gravity. Phenomenological similarities between the two approaches are studied by looking for properties predicted in one framework that are also reproducible in the other one.

First, we looked for the analogous of the URC within the MOND framework. Modifying in an observationally-based way the baryonic contribution $V_{\mathrm{bar}}$ to the rotation curve predicted by the URC, and applying the MOND formulas to this $V_{\mathrm{bar}}$, leads to a "MOND URC" whose properties are remarkably similar to the URC.

Second, it is shown that the URC predicts a tight mass discrepancy - acceleration relation, which is a natural outcome of MOND. With the choice of $V_{\text {bar }}$ that minimises the differences between the URC and the "MOND URC" the relation is almost identical to the observational one.

This similarity between the observational properties of MOND and the URC has no implications about the validity of MOND as a theory of gravity, but it shows that it can reproduce in detail the phenomenology of disk galaxies' rotation curves, as described by the URC. MOND and the URC, even though they are based on totally different assumptions, are found to have very similar behaviours and to be able to reproduce each other's properties fairly well, even with the simple assumptions made on the luminosity dependence of the baryonic contribution to the rotation curve.
\end{abstract}

Subject headings: galaxies: spiral - galaxies: kinematics and dynamics - galaxies: fundamental parameters - dark matter

\section{INTRODUCTION}

Rotation curves of spiral galaxies have been studied for several decades now, and they are a useful tool to show the mass discrepancy in galaxies: the observed kinematics and that predicted from the observed baryonic distribution do not match. Either an additional mass component or a modification of gravity are needed. In the recent past, rotation curves have been exploited to test the predictions of cosmological models of structure formation in the Universe, such as the currently favoured $\Lambda$ Cold Dark Matter $(\Lambda \mathrm{CDM})$. Most observations show that the baryons are the main kinematic component in the inner parts and that the so-called "cuspy" halos predicted by $\Lambda$ CDM (Navarro, Frenk \& White 1996, Moore et al. 1999, Navarro et al. 2004) fail to reproduce observed rotation curves (see e.g. de Blok et al. 2001; de Blok \& Bosma 2002; Gentile et al. 2004, 2005, 2007a,b; McGaugh et al. 2007).

Rotation curves have been shown to have some general properties that can be described by a stellar disk + dark matter halo model (the Universal Rotation Curve, URC; Persic, Salucci \& Stel 1996, hereafter PSS). In this framework, the circular velocity of a spiral galaxy at a certain radius depends only on one parameter, e.g. the total luminosity of the stellar disk.

MOND, the Modified Newtonian Dynamics (Milgrom 1983 ) is another successful prescription to predict the rotation curve of a spiral galaxy. According to MOND, the mass discrepancy is not due to an unseen mass compo-

\footnotetext{
${ }^{1}$ Sterrenkundig Observatorium, Ghent University, Krijgslaan 281, S9, B-9000 Ghent, Belgium

${ }^{2}$ University of New Mexico, Department of Physics and Astronomy, 800 Yale Blvd NE, Albuquerque, NM 87131, USA
}

nent (the dark matter halo), but it is instead the signature of the failure of Newtonian gravity to describe the observed kinematics at the low accelerations found in the outer parts of galaxies. In MOND, below a certain critical acceleration $a_{0}$ the Newtonian gravity is no longer valid. Earlier concerns about the inconsistency of MOND with General Relativity are now overcome with the TeVeS theory (Bekenstein 2004). MOND has a remarkable predictive power for the kinematics of galaxies: it fits the kinematics of small dwarf galaxies (Gentile et al. 2007a,c), of the Milky Way (Famaey \& Binney 2005), of early-type spiral galaxies (Sanders \& Noordermeer 2007), of massive ellipticals (Milgrom \& Sanders 2003), and it naturally explains observed tight scaling relations in spiral galaxies (McGaugh 2004, 2005).

\section{MOND}

The Modified Newtonian Dynamics (Milgrom 1983, see Sanders \& McGaugh 2002 for a review) can be invoked as an alternative to dark matter to explain the observed kinematics of disk galaxies. Within the MOND framework, the true gravitational acceleration $g$ is linked to the Newtonian one $g_{\mathrm{N}}$ through the following relation:

$$
g=\frac{g_{\mathrm{N}}}{\mu\left(g / a_{0}\right)}
$$

where $\mu(x)$ is an interpolation function whose asymptotic values are $\mu(x)=1$ when $g \gg a_{0}$ and $\mu(x)=g / a_{0}$ when $g \ll a_{0} . \quad a_{0}$ is the critical acceleration below which the Newtonian gravity is no longer valid; previous studies (Begeman, Broeils \& Sanders 1991) found that $a_{0} \sim 1.21 \times 10^{-8} \mathrm{~cm} \mathrm{~s}^{-2}$. Even though in a general case a modified version of the Poisson equation should be 
solved, eq. 2 can be shown to be a good approximation for axisymmetric disks (Brada \& Milgrom 1995). The interpolation function has been given usually the following functional form:

$$
\mu_{\text {orig }}(x)=\frac{x}{\sqrt{1+x^{2}}}
$$

However, it is obvious that a whole family of functions are compatible with the required asymptotic behaviours. For instance, Famaey \& Binney (2005) proposed that the form:

$$
\mu_{\mathrm{FB}}(x)=\frac{x}{1+x}
$$

could be a better choice, since, contrary to eq. 2 , it is compatible with the relativistic theory of MOND (TeVeS) put forward by Bekenstein (2004). Famaey et al. (2007) showed that using eq. 3 leads to a slightly different value of $a_{0}: a_{0}=1.35 \times 10^{-8} \mathrm{~cm} \mathrm{~s}^{-2}$.

If eq. 2 is used as the interpolation function, then within the MOND framework the circular velocity velocity $V_{\text {obs }}(r)$ can be expressed as a function of $a_{0}$ and the Newtonian baryonic contribution to the rotation curve $V_{\text {bar }}(r)$ at radius $r$ :

$V_{\mathrm{obs}}^{2}(r)=V_{\mathrm{bar}}^{2}(r)+V_{\mathrm{bar}}^{2}(r)\left(\sqrt{\frac{1+\sqrt{1+\left(\frac{2 r a_{0}}{V_{\mathrm{bar}}^{2}(r)}\right)^{2}}}{2}}-1\right)$

where $V_{\text {bar }}(r)=\sqrt{V_{\text {stars }}^{2}(r)+V_{\text {gas }}^{2}(r)}$ (ignoring the contribution of the bulge), $V_{\text {stars }}(r)$ and $V_{\text {gas }}(r)$ are the Newtonian contributions to the rotation curve of the stellar and gaseous disks, respectively (see Milgrom 1983). The amplitude of $V_{\text {stars }}(r)$ (whose shape is fixed by photometric observations), can be scaled according to the chosen, or fitted, stellar mass-to-light $(M / L)$ ratio. $V_{\text {gas }}(r)$ is derived from $\mathrm{HI}$ observations, when they are available. Note that the second term of the right-hand side of eq. 4 acts as a "pseudo-dark matter halo" term and that it is completely determined by the baryonic terms.

If eq. 3 is chosen as the interpolation function instead of eq. 2. the equivalent of eq. 4 becomes:

$$
V_{\mathrm{obs}}^{2}(r)=V_{\mathrm{bar}}^{2}(r)+V_{\mathrm{bar}}^{2}(r)\left(\frac{\sqrt{1+\frac{4 a_{0} r}{V_{\mathrm{bar}}^{2}(r)}}-1}{2}\right)
$$

(see e.g. Richtler et al. 2008). As expected, in both eqs. 4 and 5 the "pseudo-dark matter halo" term vanishes in the limit $a_{0} \rightarrow 0$. We note MOND has a remarkable predictive power for the general properties of rotation curves, and in many cases it is able to fit observed individual rotation curves (Kent 1987; Milgrom 1988; Begeman, Broeils \& Sanders 1991; Sanders 1996; de Blok \& McGaugh 1998). MOND correctly predicts general scaling relations linked to rotation curves, such as the baryonic Tully-Fisher relation (see McGaugh 2005, even though we note that other authors find different

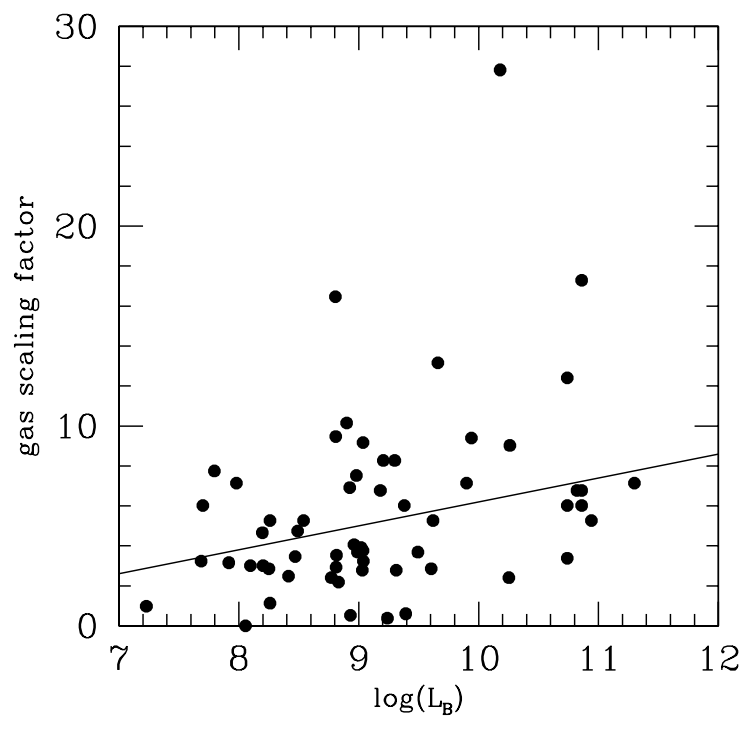

FIG. 1.- Gas scaling factor versus (log of) B-band luminosity. The data are taken from Swaters (1999) and Hoekstra et al. (2001), and the solid line is the result of a linear fit to the points.

slopes, mainly due to different choices of the stellar $M / L$ ratio) or the mass discrepancy-acceleration relation (McGaugh 2004).

\section{THE UNIVERSAL ROTATION CURVE (URC)}

PSS, from the analysis of a sample of more than 1000 rotation curves, showed that the circular velocity of a disk galaxies at a given radius (note that they considered rotation curves extended up to 2 optical radii $r_{\text {opt }}$ ) can be described as a function of only one parameter, e.g. the total luminosity. The function which describes the rotation velocity depending only on radius and luminosity is given the name Universal Rotation Curve (URC). We note that the kinematics of real galaxies depend on other factors too (such as the surface brightness), but the general phenomenological properties of rotation curves can be described well with the luminosity as only parameter.

In detail, the circular velocity $V_{\mathrm{URC}}(r)$ at radius $r$ can be expressed as a function of $V_{\mathrm{opt}}$ (the circular velocity at $r_{\mathrm{opt}}$ ), and of the stellar and dark contributions $V_{\text {disk,N }}$ and $V_{\text {halo,N }}$ normalised at $V_{\text {opt }}$ (i.e., $V_{\text {disk,N }}\left(r_{\text {opt }}\right)+$ $\left.V_{\text {halo,N }}\left(r_{\text {opt }}\right)=1\right)$ :

$$
V_{\mathrm{URC}}(r)=V_{\mathrm{opt}} \sqrt{V_{\text {disk,N }}^{2}(r)+V_{\text {halo,N }}^{2}(r)}
$$

$V_{\text {opt }}, V_{\text {disk,N }}$, and $V_{\text {halo,N }}$ can be in turn expressed as functions of $r, \lambda=L / L_{*}$ ( $L$ is the total blue luminosity of the galaxy in question and $\log L_{*}=10.4$ in solar units), and $r_{\mathrm{opt}} \simeq 13 \lambda^{0.5} \mathrm{kpc}:$

$$
\begin{gathered}
V_{\mathrm{opt}}=\frac{200 \lambda^{0.41}}{\left[0.80+0.49 \log \lambda+\frac{0.75 \exp (-0.4 \lambda)}{0.47+2.25 \lambda^{0.4}}\right]^{1 / 2}} \mathrm{~km} \mathrm{~s}^{-1} \\
V_{\text {disk }, \mathrm{N}}^{2}=(0.72+0.44 \log \lambda) \frac{1.97\left(r / r_{\mathrm{opt}}\right)^{1.22}}{\left[\left(r / r_{\mathrm{opt}}\right)^{2}+0.61\right]^{1.43}}
\end{gathered}
$$




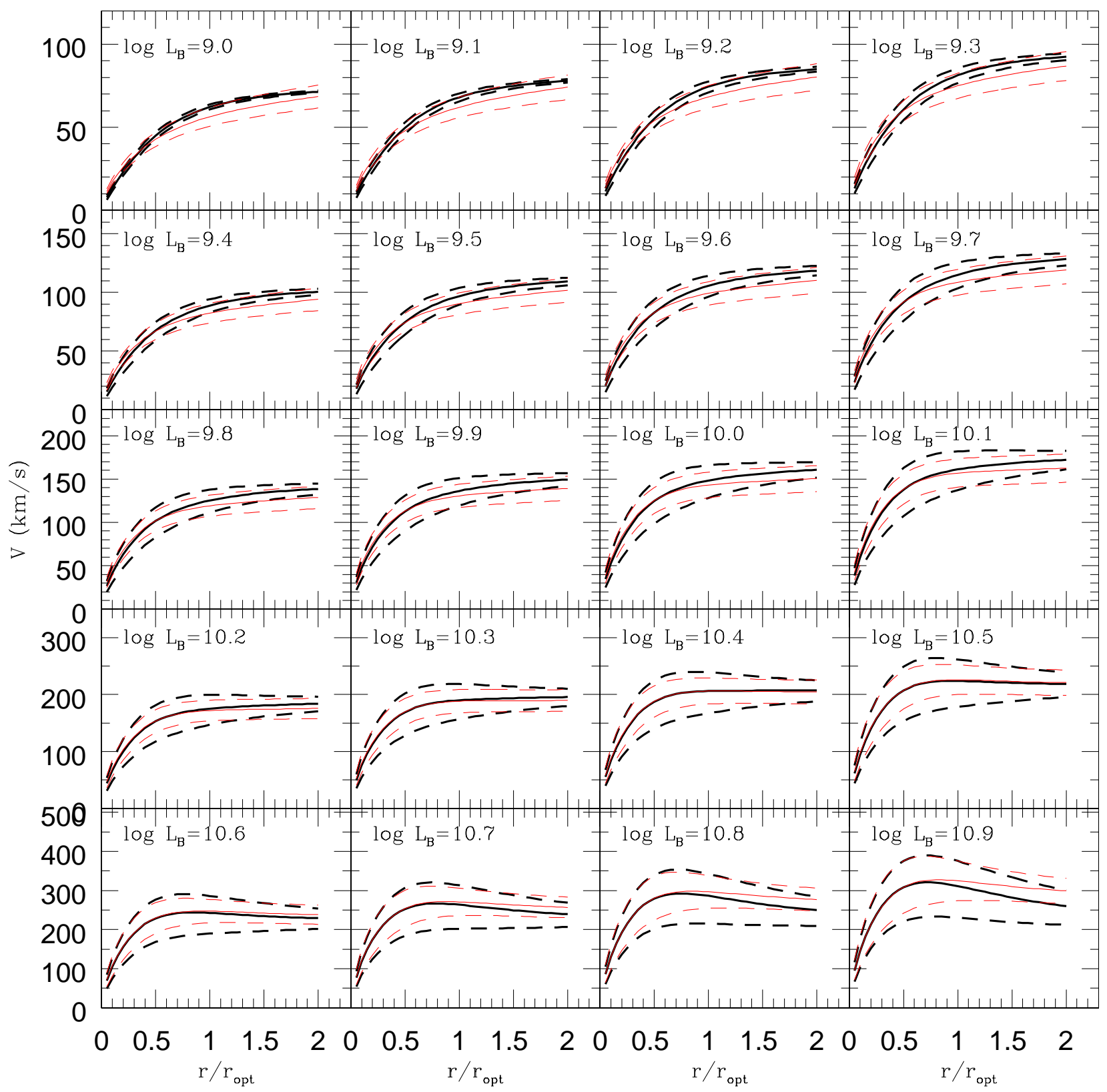

FIG. 2.- Comparison between the Universal Rotation Curve (thick black curves), at various luminosities, and the equivalent for MOND (thin red curves, using $\mu_{\text {orig }}(x)$ ), with the stars and gas contributions defined in the text. Dotted curves indicate the uncertainties.

$$
V_{\text {halo, } \mathrm{N}}^{2}=1.6 \exp (-0.4 \lambda) \frac{\left(r / r_{\mathrm{opt}}\right)^{2}}{\left(r / r_{\mathrm{opt}}\right)^{2}+2.25 \lambda^{0.4}}
$$

According to PSS, eqs. 6 to 9 predict the circular velocity of a spiral galaxy at radius $r$ with an uncertainty of $4 \%$. Choosing $r_{\mathrm{opt}} \simeq 13 \lambda^{0.5} \mathrm{kpc}$ hides any variation of surface brightness for a given luminosity, which results in different rotation curve shapes for galaxies with different surface brightnesses (see e.g. McGaugh \& de Blok 1998). However, we note that the original formulation of the URC (with the radius $r$ in units of the stellar exponential scale length $r_{\mathrm{D}}$ ) takes this effect into account, since the rotation curves of low- and high-surface brightness galaxies are virtually indistinguishable once the radius is expressed in units of $r_{\mathrm{D}}$ (e.g., Verheijen \& de Blok 1999). Also, in the URC the contribution of the gaseous disk is ignored. This is acceptable in the range of luminosities considered by PSS because a) the gasous disk never dominates the kinematics and b) because of its scaling with the dark matter contribution, its contribution can be implicitely accounted by $V_{\text {halo }}$.

An important feature of the URC is the strong dependence of the logarithmic slope of the rotation curve $\nabla$ on the luminosity. They define $\nabla$ in the range $0.5 r_{\text {opt }}<$

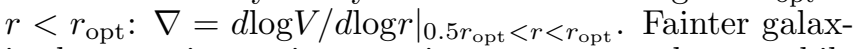
ies have an increasing rotation curve around $r_{\text {opt }}$, while in the most massive galaxies $V(r)$ is already decreasing around $r_{\mathrm{opt}}$.

\section{THE MOND UNIVERSAL ROTATION CURVE}

The question that this paper is addressing is: since both MOND and the URC are successful ways to predict the general kinematical properties of disk galaxies, 


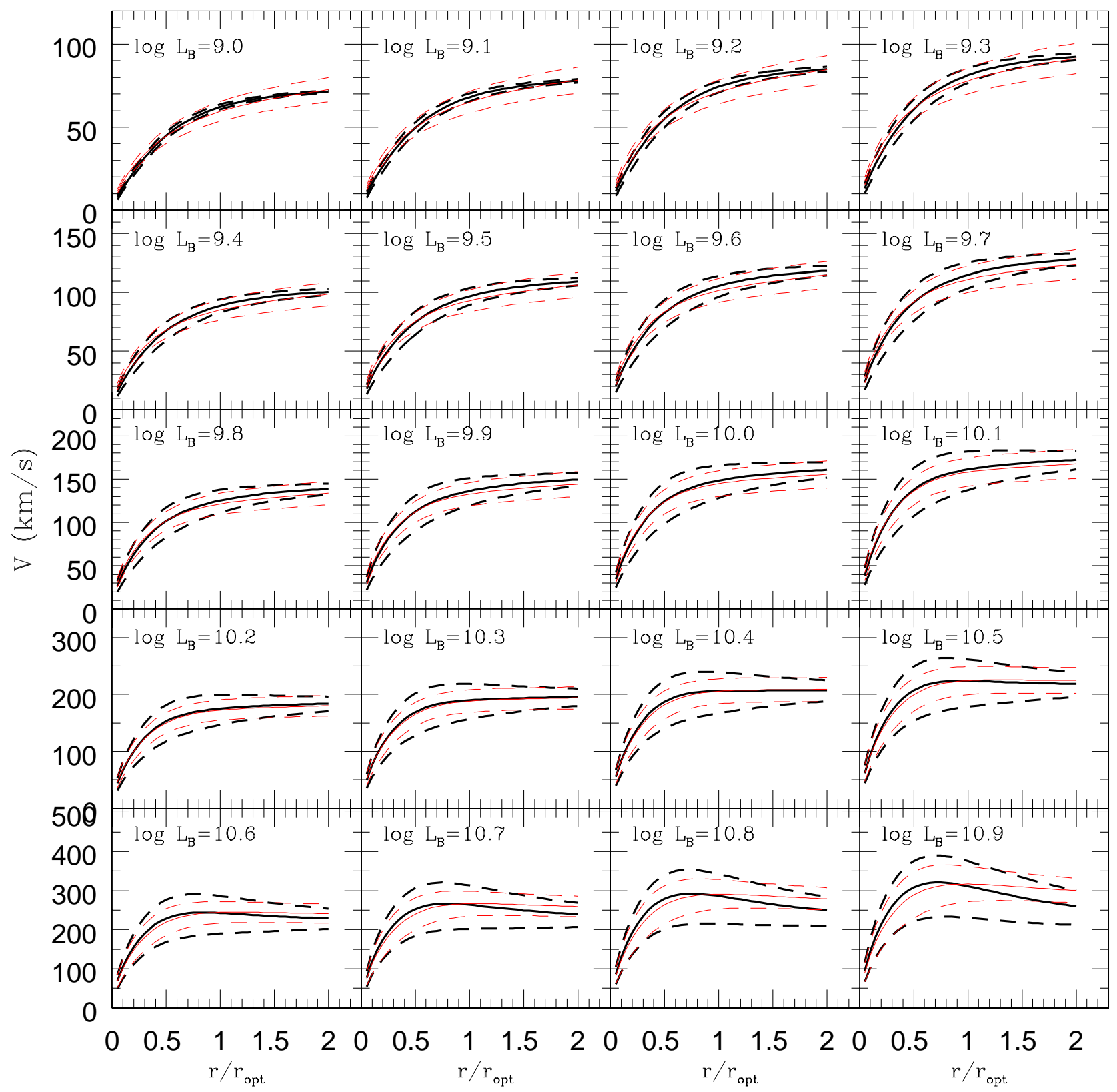

FIG. 3.- Comparison between the Universal Rotation Curve (thick black curves), at various luminosities, and the equivalent for MOND (thin red curves, using $\mu_{\mathrm{FB}}(x)$ ), with the stars and gas contributions defined in the text. Dotted curves indicate the uncertainties.

to what extent do they have similar properties? One way to compare their properties is to try to derive something like the URC within the framework of MOND. In order to achieve this, both $V_{\text {stars }}(r)$ and $V_{\text {gas }}(r)$ are needed. For the former term, the URC provides us with a recipe to estimate it, while the latter is ignored in the URC. A rough estimate of $V_{\text {gas }}(r)$ can be made through the scaling property of $V_{\text {halo }}(r)$ compared to $V_{\text {gas }}(r)$ noticed by e.g. Bosma (1981) and Hoekstra, van Albada \& Sancisi (2001): $V_{\text {halo }}^{2}(r) \approx n \times V_{\text {gas }}^{2}(r)$. The factor $n$ was calculated by performing a linear fit to the observational data in the scaling factor versus luminosity plot (see Fig. 1). The data were taken from the samples of Swaters (1999) and Hoekstra et al. (2001). The former work gives Rband luminosities, whereas the URC uses B-band. The B-band luminosities of the Swaters (1999) sample were calculated from the LEDA database, when available, and from NED. The linear fit gives $n=1.3 \log \lambda-6.4$. Therefore, we estimated $V_{\text {gas }}(r)$ through the following relation: $V_{\text {gas }}(r) \sim V_{\text {halo, URC }}(r) / \sqrt{1.3 \log \lambda-6.4}$. This turns out to be in line with the findings of Swaters (1999), who finds $n$ to be correlated with surface brightness, and hence with luminosity. Such a scaling of the actual surface density with the gas surface density would happen in the case of a conspicuous amount of disk dark matter, e.g. $\mathrm{H}_{2}$ in the form of clumps (Pfenniger, Combes \& Martinet 1994) or in a cold neutral medium phase (Papadopoulos, Thi \& Viti 2002).

The MOND formulas (eqs. 4 and 5) were then applied to the $V_{\text {stars }}(r)$ and $V_{\text {gas }}(r)$ derived above. However, using the face value $V_{\text {stars }}(r)$ coming from the URC formulas leads to a MOND Universal Rotation Curve that is quite different from the original URC, especially for galaxies where the stellar disk dominates the kinematics. 
In the lower panels of Figs. 2 and 3 the MOND curves would lie significantly above the URC curves. Thus, a parameter $\eta$ such that $V_{\text {stars,MOND }}^{2}(r)=\eta V_{\text {stars, URC }}^{2}(r)$ was fitted in order to have the best possible agreement between the URC and the "MOND URC", assuming that the scaling of the stellar mass-to-light ratio with luminosity predicted by the URC is correct.

This parameter $\eta$ corresponds to the ratio of the stellar $\mathrm{M} / \mathrm{L}$ ratios required in MOND and the URC frameworks, respectively. A best-fit value of $\eta=0.77$ was found for the standard interpolation function (eq. 2) and a value of $\eta=0.57$ for the simple one (eq. 3). This is in agreement with Famaey et al. (2007), who compare rotation curve fits made with the two $\mu$ functions considered here are conclude that the simple $\mu$ function gives stellar $M / L$ ratios that are on average $\sim 30 \%$ lower than with the standard $\mu$.

The stellar mass-to-light ratios are generally not well known in spiral galaxies. Here we considered a conservative estimate of the uncertainties to be $50 \%$ (see de Jong \& Bell 2006). The uncertainty on the gas scaling factor $n$ was taken to be 3.4 , from the scatter in the distribution of $n$ shown in Fig. 1. This scatter is large, but it is not possible to find more a accurate way to estimate the scaling factor within the context of the URC, because one would need to consider other parameters (e.g. the Hubble type) than the luminosity, which are much more difficult to take into account: for instance, in early-type disk galaxies usually there is a central depression in the $\mathrm{HI}$ distribution, but its size and magnitude are variable. Moreover, the URC, by construction, has the luminosity as the only parameter varying from galaxy to galaxy. The uncertainties on the baryonic contribution to the rotation curve result in uncertainties on the calculated rotation curves. They are only rough estimates, since other (less easily quantifiable) sources of errors were ignored, such as the presence of the bulge. These uncertainties on the stellar $M / L$ ratios mean that the disagreement between the MOND and URC stellar $M / L$ ratios (i.e., $\eta$ being $\neq 1$ ) does not necessarily imply that the approach of the present paper is incorrect. Moreover, this is in qualitative agreement with the fact that 1) in McGaugh (2005) the ratio of the "maximum" $M / L$ ratio and the MOND $M / L$ ratio has a median value of 1.8 , where "maximum" in McGaugh (2005) is admittedly loosely defined but it is generally taken as a fit where the peak of the rotation curve can be entirely explained by the stellar disk; and 2 ) the URC has disks close to "maximum" (following the above definition) for most galaxies apart from the least massive ones.

The URC and the "MOND URC" (using $\mu_{\text {orig }}(x)$ ) are compared in Fig. 2. for the whole range of circular velocities and radii usually sampled by rotation curves, the two datasets display almost identical properties. Not surprisingly, the best agreement is found for the range $100 \mathrm{~km} \mathrm{~s}^{-1} \lesssim V_{\text {opt }} \lesssim 250 \mathrm{~km} \mathrm{~s}^{-1}$, where most observed rotation curves lie. The worst disagreement is found for the largest distances, in particular in the most massive galaxies. This has to be expected, though, as the number of data points in this region of the parameter space is quite small: the sample of PSS has very few data for large radii of massive galaxies. Indeed, only a minority of the curves come from HI observations (which trace the

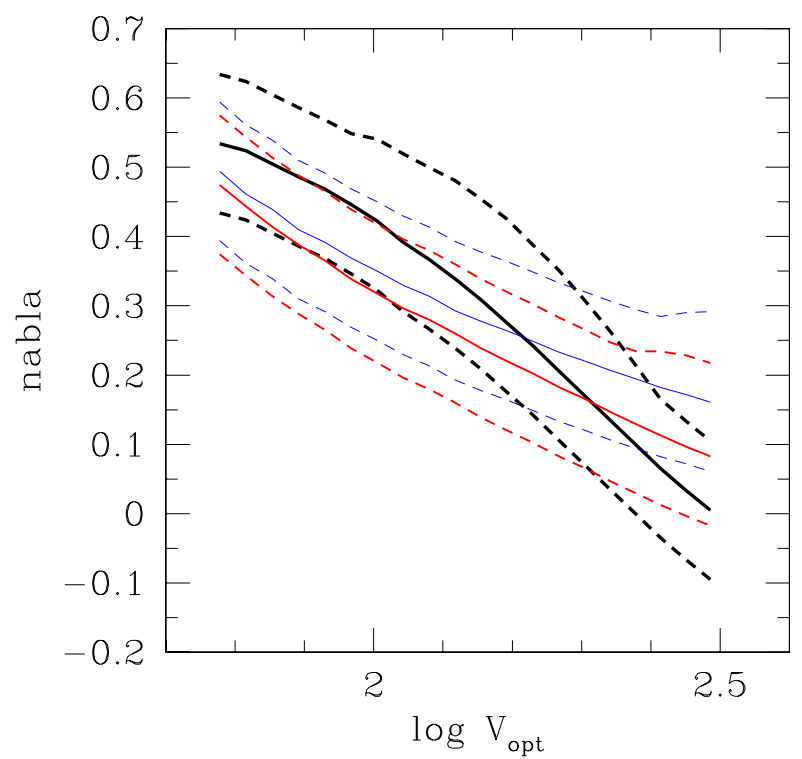

FIG. 4.- Logarithmic slope of the rotation curve versus $\log \left(V_{\text {opt }}\right)$. The thick black lines represents the URC, the (less thick) red and (thin) blue lines represent the MOND curves, with the standard and simple $\mu$ functions, respectively.

kinematics typically out to 2-3 $r_{\text {opt }}$ ), and few galaxies are early-type large spirals (only two galaxies in their sample have type Sab or earlier). However, using the uncertainties on the baryonic contribution discussed above, the URC and the MOND URC are always in agreement with each other within the errorbars. Also, note that in this paper we ignored the contribution of the bulge, since it was ignored in the URC. This is likely to have a strong effect on the rotation curves of the largest galaxies.

The same considerations can be made if one uses $\mu_{\mathrm{BF}}(x)$ instead of $\mu_{\mathrm{orig}}(x)$. Fig. [3 shows that the URC and the "MOND URC" using $\mu_{\mathrm{BF}}(x)$ agree with each other as well as if one uses $\mu_{\text {orig }}(x)$.

Using either interpolation function, the trend in logarithmic slope (see Section 3) is also reproduced: in the outer parts of the galaxy, the faintest galaxies have a rising rotation curve while the brightest galaxies have a declining rotation curve. This is illustrated in Fig. 4, where the logarithmic slope as a function of $V_{\text {opt }}$ is displayed. Again, within the uncertainties the two formalisms agree with each other. MOND does not reproduce particularly well the slopes of the most massive galaxies, mainly because of the fact that the bulge is not taken into account. For consistency with PSS we first performed a linear fit of the rotation curves between 0.5 and $1 r_{\mathrm{opt}}$, then we took the slope at $1 r_{\mathrm{opt}}$. The uncertainties on the slopes were computed as the maximum between the propagation of the uncertainties on the rotation curves and a minimum value of 0.1 (see PSS).

\section{THE MASS DISCREPANCY-ACCELERATION RELATION FOR THE URC}

Now that it has been shown that MOND can reproduce something which is quite close to the URC, in order to further compare the two phenomena let us have a look if the URC can reproduce one of the main properties linked to MOND: the mass discrepancy-acceleration relation. McGaugh (2004) showed that rotation curve data seem to organise themselves along a relation linking 
the mass discrepancy (defined as $D(r)=V^{2}(r) / V_{\mathrm{bar}}^{2}(r)$, where $V(r)$ is the rotation velocity at radius $r$ ) to the gravitational acceleration (here the Newtonian acceleration is used, $\left.g_{\mathrm{N}}=V_{\mathrm{bar}}^{2} / r\right)$. In the same paper, it is also shown that the stellar $M / L$ ratios arising from the MOND fits minimise the scatter in the mass discrepancyacceleration relation.

Fig. 5 shows that using the URC formulas one finds such a relation, whatever choice of baryonic mass is made. Since we are looking for such a relation in the URC context, the mass discrepancy is always computed with respect to the URC rotation curve (i.e., in the above definition of $\left.D(r), V^{2}(r)=V_{\mathrm{URC}}^{2}(r)\right)$. Obviously, using $V^{2}(r)=V_{\mathrm{MOND}}^{2}(r)$ (whichever $\mu$ is used) leads to a zeroscatter relation, by construction. A priori the tightness of the relations shown in Fig. 5 comes a bit as a surprise: while in MOND a critical acceleration is inbuilt in the theory, in the URC framework there is no such thing, at least in an explicit way. Semi-analytical dark matter models (e.g., van den Bosch \& Dalcanton 2000) can also reproduce a mass discrepancy-acceleration relation using the right choice of parameters. However, the main thing to note is that in the present paper the URC gives a mass discrepancy-acceleration relation without any parameter to adjust.

From the lower four panels Fig. [5] one can see that the mass discrepancy-acceleration relation from the URC formulas is consistent with that found by McGaugh (2004) with observational data if the baryonic contribution to the rotation curve is chosen to be the same as in Section 4, with $\eta=0.57$ or 0.77 . The former value of $\eta$ slightly overestimates the mass discrepancy (compared to McGaugh's points) for a given Newtonian acceleration $g_{\mathrm{N}}$ : this is to be expected because $\eta=0.57$ corresponds to the "simple" interpolation function, where the full MOND regime is reached faster than with the "standard" function. Using $\eta=0.77$ gives an excellent agreement: it corresponds to the "standard" interpolation function, which is what McGaugh used. Taking the face-value $V_{\mathrm{bar}}(r)$ predicted by the URC yields a remarkably tight relation; the scaling is obviously different, since the stellar $M / L$ ratio is different and the gaseous contribution is ignored. We also considered different ranges of luminosities: since the URC is defined for $V_{\mathrm{opt}} \gtrsim 80$ $\mathrm{km} \mathrm{s}^{-1}$ (see PSS), the predicted scaling relations are not expected to hold with high accuracy. Indeed, the left panels Fig. [5] show that for $V_{\text {opt }} \lesssim 80 \mathrm{~km} \mathrm{~s}^{-1}$ the innermost few points of the rotation curves start to become discrepant.

\section{DISCUSSION}

One of the main differences between the URC and the MOND fits is the stellar $M / L$ ratio: while in the former disks are mostly close to maximum (following the definition in Section (4), the latter yields slightly submaximal disks (see McGaugh 2005), which results in a very different scaling in the mass discrepancy - acceleration plane. Again, we note that a limitation of the present approach is that these statements ignore any surface brightness variation at equal luminosity. A robust, precise and incontrovertible method to determine stellar $M / L$ ratios is yet to be found. A number of methods have been suggested (see e.g. de Jong \& Bell 2006), which suggest $M / L$ ratios ranging roughly between half-maximum to maxi- mum disks. Therefore, a distinction between the URC and MOND based on the predicted stellar $M / L$ ratios is not viable at the moment. The fact that the simple $\mu$ function gives lower stellar $M / L$ than the standard one has already been shown by Famaey et al. (2007).

In the present paper the discussion is focussed only on the general properties of spiral galaxies' rotation curves. Both MOND and the URC are successful ways of describing the overall behaviour of rotation curves, but inevitably there are cases where they fail to reproduce the details in the rotation curves. Also, only a rough estimate of $V_{\mathrm{bar}}(r)$ was made in the context of MOND, in particular for $V_{\text {gas }}(r)$. The observed $V_{\text {halo }}(r) / V_{\text {gas }}(r)$ scaling shows considerable variations around the value of $\sqrt{1.3 \log \lambda-6.4}$ that was assumed here. Despite these large approximations, the URC and MOND exhibit remarkable phenomenological similarities.

A major feature of MOND and the URC is that in both frameworks one is able to infer in a reasonably accurate way the general properties of rotation curves based on the amount and distribution of baryonic matter only. This is closely linked to another property of rotation curves (Sancisi 2003), whereby for any feature in a rotation curve there is a corresponding feature in the baryonic distribution. The literature is full of such examples, one of the most spectacular being NGC 1560 (Broeils 1992). This empirical fact is unexpected in a Cold Dark Matter (CDM) framework since CDM fits to the rotation curves require strongly submaximal disks, which would cancel this baryon-kinematics coupling that is observed (see e.g. de Blok et al. 2001, McGaugh 2004, Gentile et al. 2004). Yet another evidence of the close coupling of baryonic and general properties of spiral galaxies is the observed correlation between stellar exponential scale lengths and halo core radii (Donato, Gentile \& Salucci 2004). A similar effect can be inferred also within MOND (Milgrom \& Sanders 2005). The interpretation of the baryon-kinematics coupling is not obvious: possible solutions are that baryons and dark matter are interacting in some unknown way other than gravity in the central parts of galaxies or that a MOND-like theory such as $\mathrm{TeVeS}$ is the correct theory of gravity.

A possible concern about the approach presented in this paper might have been that the similarity between MOND and the URC might actually be an identity. This is not the case, since even if one assumes the same baryonic contribution in both frameworks (e.g. the ones considered in Section 4, with either $\eta=0.57$ or 0.77 ), the functional form of the halo contribution in the URC context (eq. 9) is completely different from the functional form of the "pseudo-halo" contribution in MOND (second term of the right-hand side of eq. (4).

As pointed out by Salucci \& Gentile (2006), any alternative theory of gravity (such as MOND or MOG, see Moffat 2006) should be able to account for the observed phenomenology of rotation curves. In the present paper we have demonstrated that MOND does fairly well, despite the naive expectation of having only flat rotation curves because of the asymptotically flat behaviour of the MOND rotation curves. Hence, the MOND framework not only usually gives good fits to rotation curves, but it also predicts the correct scaling of the rotation curves properties with luminosity, as shown by the URC. 
$\log \mathrm{L} / \mathrm{L}_{*}: 9.0 \rightarrow 10.9$
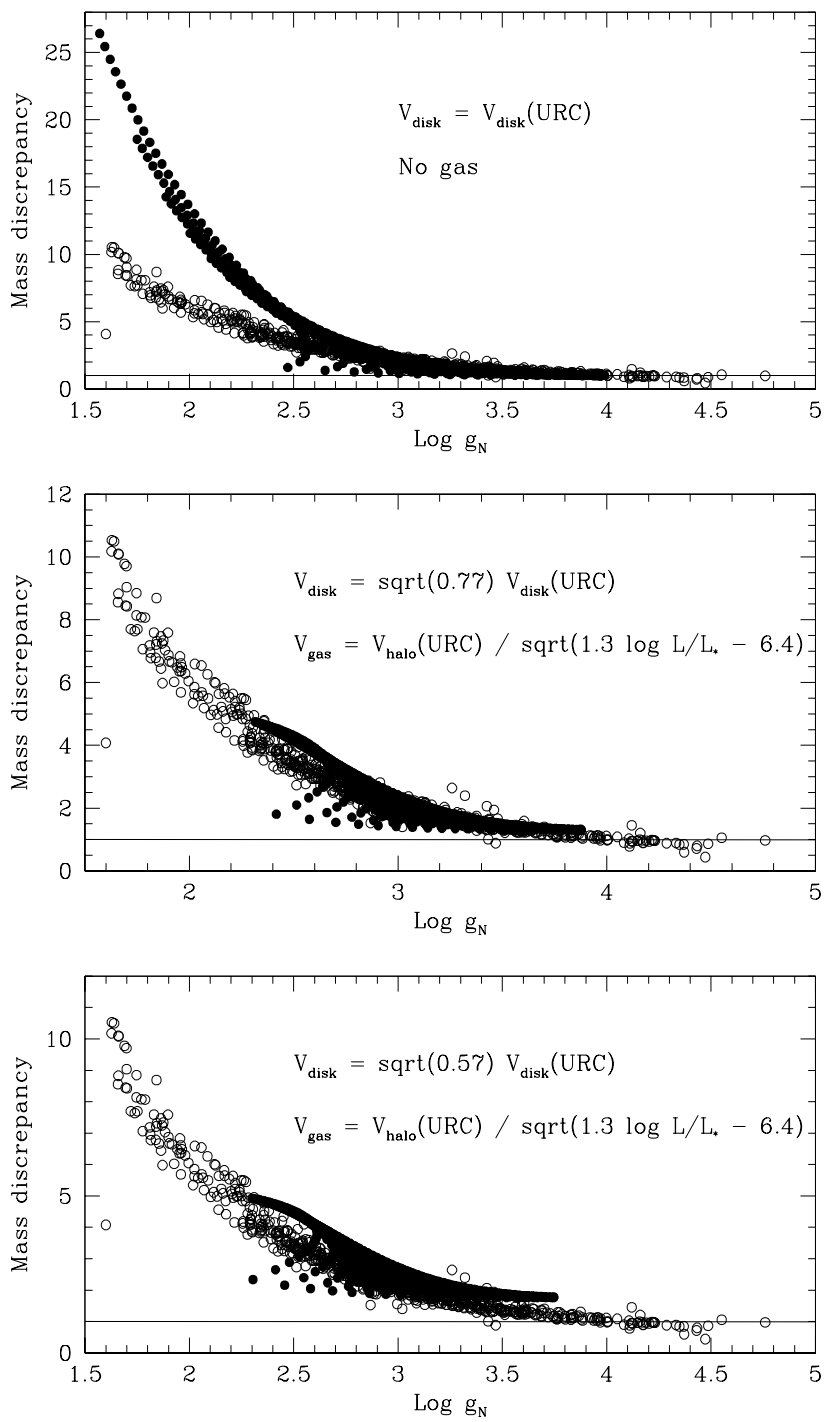

$\log \mathrm{L} / \mathrm{L}_{*}: 9.3 \longrightarrow 10.9$
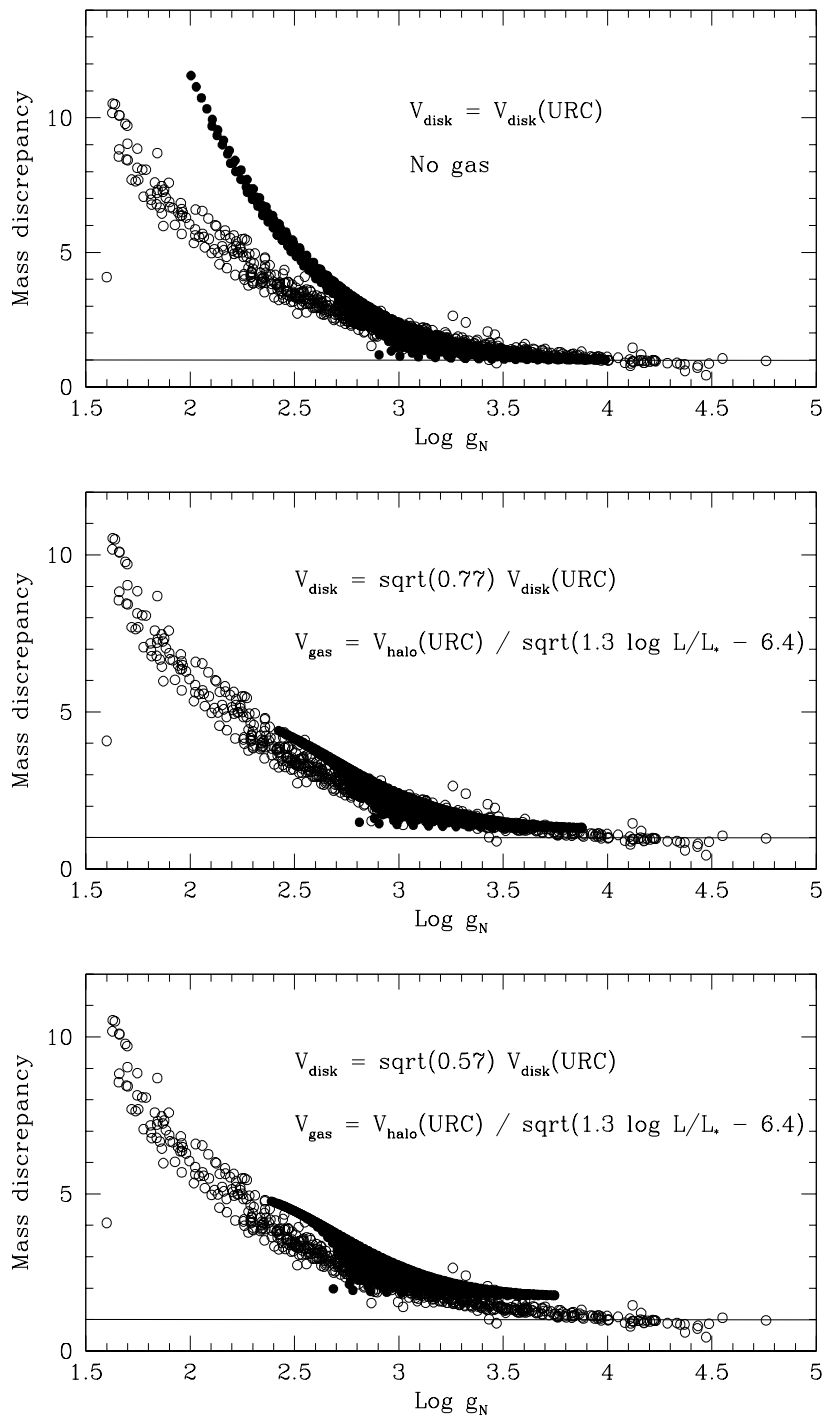

FIG. 5.- Full circles: mass discrepancy-acceleration (MDA) relation using the URC formulas. Open circles: the MDA data from McGaugh (2004), using the stellar M/L ratios arising from the MOND rotation curve fits. The horizontal axis is the log of the Newtonian acceleration (in units of $\mathrm{km}^{2} \mathrm{~s}^{-2} \mathrm{kpc}^{-1}$ ) and the mass discrepancy is defined with respect to the URC rotation curve. The ranges of B-band luminosities are indicated at the top (in solar units) and the radii range from $0.05 r_{\mathrm{opt}}$ to $2 r_{\mathrm{opt}}$, in steps of $0.05 r_{\mathrm{opt}}$. In the upper panel the face-value URC prescription for the baryons was considered, while for the middle and bottom panel the values for $V_{\text {disk }}$ and $V_{\text {gas }}$ were taken as in Section 4

\section{CONCLUSIONS}

Starting from the consideration that both MOND and the Universal Rotation Curve (URC) are valid ways to describe the general properties of rotation curves, the two approaches were compared by trying to reproduce one's predictions using the prescriptions of the other one.

The first comparison was made by attempting to reproduce something like the URC in the MOND framework. Some (observationally-based) assumptions on the stellar and gaseous contributions to the rotation curve were made. It turns out that it is possible to build a "MOND URC" with very similar properties to the URC. The MOND URC also exhibits the trend in logarithmic slope versus luminosity seen in the URC. Both the "standard" MOND interpolation function and the simple one proposed by Famaey \& Binney (2005) were tested, with almost identical results.
The second comparison was made by looking for a mass discrepancy - acceleration relation using the URC formulas. With all three choices of baryonic contributions $\left(V_{\text {bar }}\right)$ considered in this paper a tight (unexpected a priori) relation arises. Using the $V_{\text {bar }}$ values that match best the "MOND URC" to the URC one finds a mass discrepancy - acceleration relation like the observed one.

While these results have no implications as to whether MOND is a valid theory of gravity, MOND and the URC, even though they are based on totally different assumptions, are found to display similar properties and to reproduce each other's predictions well.

I wish to thank the referee for constructive comments that improved the quality of the paper, and Stacy McGaugh for providing his data on the mass discrepancy acceleration relation. GG is a postdoctoral fellow with 
the National Science Fund (FWO-Vlaanderen).

\section{REFERENCES}

Begeman, K. G., Broeils, A. H., Sanders, R. H., 1991, MNRAS, 249,523

Bekenstein, J. D., 2004, Phys. Rev. D., 70, 083509

Bosma, A., 1981, AJ, 86, 1791

Brada, R., \& Milgrom, M., 1995, MNRAS, 276, 453

Broeils, A. H., 1992, A\&A, 256, 19

de Blok, W. J. G., McGaugh, S. S. 1998, ApJ, 508, 132

de Blok, W. J. G., McGaugh, S. S., Rubin, V. C., 2001, AJ, 122 2396

de Blok, W. J. G., Bosma, A., 2002, A\&A, 385, 816

de Jong, R. S., Bell, E. F., 2006, preprint, astro-ph/0604391

Donato, F., Gentile, G., Salucci, P. 2004, MNRAS, 353, L17

Famaey, B., Binney, J., 2005, MNRAS, 363, 603

Famaey, B., Gentile, G. , Bruneton, J.-P., Zhao, H. S., 2007, Phys. Rev. D, 75, 063002

Gentile, G., Salucci, P., Klein, U., Vergani, D., Kalberla, P., 2004, MNRAS, 351, 903

Gentile, G., Burkert, A., Salucci, P., Klein, U., Walter, F., 2005, ApJ, 634, L145

Gentile, G., Salucci, P., Klein, U., Granato, G. L. 2007a, MNRAS, 375, 199

Gentile, G., Tonini, C., Salucci, P., 2007b, A\&A, 467, 925

Gentile, G., Famaey, B., Combes, F., Kroupa, P., Zhao, H. S., Tiret, O. 2007c, A\&A, 472, L25

Hoekstra, H., van Albada, T. S., Sancisi, R., 2001, MNRAS, 323, 453

Kent, S. M. 1987, AJ, 93, 816

McGaugh, S. S., de Blok, W. J. G. 1998, ApJ, 499, 41

McGaugh, S. S., 2004, ApJ, 609, 652

McGaugh, S. S., 2005, ApJ, 632, 859
McGaugh, S. S., de Blok, W. J. G., Schombert, J. M., Kuzio de Naray, R., Kim, J. H. 2007, ApJ, 659, 149

Milgrom, M., 1983, ApJ, 270, 365

Milgrom, M. 1988, ApJ, 333, 689

Milgrom, M., Sanders, R. H. 2003, ApJ, 599, L25

Milgrom, M., Sanders, R. H. , 2005, MNRAS, 357, 45

Moffat, J. W. 2006, Journal of Cosmology and Astro-Particle Physics, 3, 4

Moore, B., Quinn, T., Governato, F., Stadel, J., Lake, G., 1999, MNRAS, 310, 1147

Navarro, J. F., Frenk, C. S., White, S. D. M., 1996, ApJ, 462, 563

Navarro, J. F., Hayashi, E., Power, C., Jenkins, A. R., Frenk, C. S., White, S. D. M., Springel, V., Stadel, J., Quinn, T. R., 2004, MNRAS, 349, 1039

Papadopoulos, P. P., Thi, W.-F., \& Viti, S. 2002, ApJ, 579, 270

Persic, M., Salucci, P., Stel, F., 1996, MNRAS, 281, 27

Pfenniger, D., Combes, F., \& Martinet, L. 1994, A\&A, 285, 79

Richtler, T., Schuberth, Y., Hilker, M., Dirsch, B., Bassino, L., \& Romanowsky, A. J. 2008, A\&A, 478, L23

Salucci, P., Gentile, G. 2006, Phys. Rev. D, 73, 128501

Sancisi, R. 2003, in IAU Symp. 220, Dark Matter in Galaxies (Dordrecht: Kluwer), 192

Sanders, R. H. 1996, ApJ, 473, 117

Sanders, R. H., McGaugh, S. S., 2002, ARA\&A, 40, 263

Sanders, R. H., Noordermeer, E. 2007, MNRAS, 379, 702

Swaters, R. A. 1999, Ph.D. Thesis, University of Groningen

Verheijen, M., de Blok, E. 1999, Ap\&SS, 269, 673 